\title{
Hydrodynamic analysis of a floating body with an open chamber using a 2D fully nonlinear numerical wave tank
}

\author{
Ahmed Syed Uzair and Weoncheol Koo \\ School of Naval Architecture and Ocean Engineering, University of Ulsan, Korea
}

\begin{abstract}
Hydrodynamic analysis of a surface-piercing body with an open chamber was performed with incident regular waves and forced-heaving body motions. The floating body was simulated in the time domain using a 2D fully nonlinear numerical wave tank (NWT) technique based on potential theory. This paper focuses on the hydrodynamic behavior of the free surfaces inside the chamber for various input conditions, including a two-input system: both incident wave profiles and forced body velocities were implemented in order to calculate the maximum surface elevations for the respective inputs and evaluate their interactions. An appropriate equivalent linear or quadratic viscous damping coefficient, which was selected from experimental data, was employed on the free surface boundary inside the chamber to account for the viscous energy loss on the system. Then a comprehensive parametric study was performed to investigate the nonlinear behavior of the wave-body interaction.
\end{abstract}

KEY WORDS: Numerical wave tank; Open chamber; Viscous damping; Wave-body interaction; Chamber surface elevation, Oscillating water column.

\section{INTRODUCTION}

There has been a rapid development in energy extraction devices from renewable energy sources in the past few decades. Advancements in renewable energy technology have reduced the cost and increased the reliability and confidence of using such devices. Water waves have emerged as a promising source of renewable energy. In fact, it is easy to visualize the energy of these waves as they forcefully splash against natural and artificial sea walls.

Accordingly, numerous wave energy converters have been proposed, developed, and tested. One of the most promising concepts is the oscillating water column (OWC) device that utilizes specially designed air turbines to achieve pneumatic power take off; examples include the Wells turbine, impulse turbine, and Dennis-Auld turbine. Since Masuda and Miyazaki (1978) first proposed and tested the OWC concept, several commercial-level OWC plants have been constructed and successfully operated. One such prototypical OWC plant is the Land Installed Marine Powered Energy Transformer (LIMPET) located on the Isle of Islay in Scotland (Heath, Whittaker and Boake, 2000).

In this paper, the nonlinear wave-body interaction of a floating body with an open chamber, which is a feasible floating OWC structure, is studied using a 2D fully nonlinear numerical wave tank (NWT) technique. The utilized NWT is based on potential fluid theory and the boundary element method (BEM) with constant panel discretization. This study also uses a mixed Eulerian-Lagragian (MEL) free surface treatment and Runge-Kutta fourth-order (RK4) time-integration techniques, along with a smoothing scheme on the free surface. A specially optimized $\phi_{n}-\eta$ artificial damping scheme is employed on the free surface to minimize the wave reflection from the end wall and re-reflection from the input boundary.

Corresponding author: Weoncheol Koo

e-mail:wckoo@ulsan.ac.kr 
This work presents a time-domain analysis of the hydrodynamic behavior of the surface elevation in the gap of a floating body with two input systems. Several researchers have considered a similar system with an open-chamber-type surface-piercing body, including Lin, Newman and Yue (1984), Faltinsen, Rognebakke and Timokham (2007), Koo (2009), and Yong and Mian (2010). However, their works were conducted using only one input e.g., modeling a diffraction problem with incident waves only, or employing forced-body motions to generate radiation waves. In this study, the numerical analysis of such a structure is extended to incorporate two inputs: the incident waves and the forced-heave velocity of the body. This analysis allows us to investigate the effect of the respective inputs and to find the optimal input conditions for the maximum mid-chamber free surface elevation, which can then be used to optimize the design of a floating OWC wave energy converter. Nonlinear timedomain results are compared with the linear results to inspect the nonlinearities of the solutions and the effectiveness of a linear calculation.

\section{MATHEMATICAL FORMULATIONS}

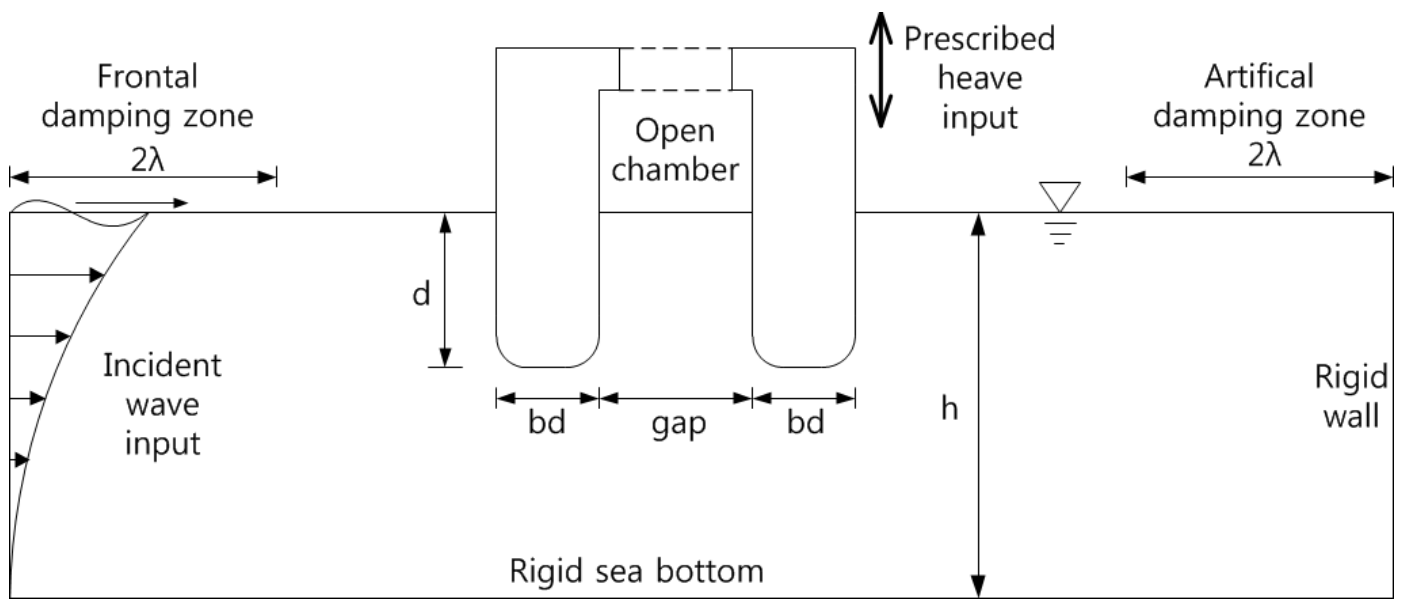

Fig. 1 Overview of the computational domain of a floating body with an open chamber, where $\mathrm{h}=1.03 \mathrm{~m}, \mathrm{~d}=0.18 \mathrm{~m}, \mathrm{bd}=0.36 \mathrm{~m}$, gap $=0.18 \mathrm{~m}$, and corner radius $=0.01 \mathrm{~m}$.

In order to analyze the hydrodynamic behavior of a free surface, the mixed boundary value problem can be solved in the computational fluid domain. Fig. 1 shows the overview of a floating body with an open chamber in the computational domain. Assuming an inviscid, irrotational, and incompressible fluid, the Laplace equation can be satisfied as a governing equation in the potential-fluid domain:

$$
\nabla^{2} \phi=0
$$

Then, the fully nonlinear dynamic and kinematic free surface boundary conditions can be given respectively as

$$
\frac{\partial \phi}{\partial t}=-g \eta-\frac{1}{2}|\nabla \phi|^{2}-\frac{P_{a}}{\rho}, \quad \frac{\partial \eta}{\partial t}=-\nabla \phi \cdot \nabla \eta+\frac{\partial \phi}{\partial z}
$$

where $\eta$ is free surface elevation and $P_{a}$ is the air pressure, which is set to zero on the free surface.

As the boundary condition for the rigid surfaces, including the fixed body surface, the sea bottom, and the vertical end-wall, the normal flux was set to zero:

$$
\frac{\partial \varphi}{\partial n}=0
$$


As an input boundary condition, an incident wave can be generated by two different methods: (i) by manually moving the wave maker as prescribed, or (ii) by feeding analytic linear and nonlinear waves (i.e., Stokes' $2^{\text {nd }}$-order) along the vertical input boundary. The incident wave for the present study was generated by feeding second-order Stokes waves along the left side of the domain. These waves can be described as

$$
\begin{aligned}
\frac{\partial \phi}{\partial n}=n_{x} \frac{\partial \phi}{\partial x} & =n_{x} \frac{g A k}{\omega} \frac{\cosh k(z+h)}{\cosh k h} \cos (k x-\omega t) \\
& +n_{x} \frac{3}{8} A^{2} \omega(2 k) \frac{\cosh 2 k(z+h)}{\sinh ^{4} k h} \cos 2(k x-\omega t)
\end{aligned}
$$

where $\mathrm{A}, \omega, \mathrm{k}$, and $\mathrm{h}$ represent the wave amplitude, frequency, wave number, and water depth, respectively, and $n_{x}$ denotes the normal vector in the $\mathrm{x}$-direction.

Next, the body-input boundary condition can be expressed such that the fluid particle velocity on the body surface equals the body velocity in the normal direction:

$$
\frac{\partial \phi}{\partial n}=V_{B} \cdot \vec{n}
$$

where $\vec{n}$ is the normal vector and $V_{B}$ is the body velocity with respect to the center of gravity. In the present study, the prescribed body velocity $V_{B}$ can be applied to the system as a body input $\left(V_{B}=\eta_{b} \cos (\omega t)\right.$ for the heaving velocity, where $\eta_{b}$ is the heaving amplitude of the body). Note that when the body is stationary, the body boundary condition is the same as Eq. (3).

In order to solve the governing equation associated with the given boundary conditions, the Laplace equation should be transformed into a boundary integral equation (see Eq. (6)) using a Green function. Discretizing the entire boundary and placing a node on each boundary element, which is a process called the constant panel method (CPM), the boundary integral equation can be decomposed into a series of equations in matrix form. These equations can then be solved numerically at each time step to obtain the velocity potential and its normal derivative at each nodal point.

$$
\alpha \phi_{i}=\iint_{\Omega}\left(G_{i j} \frac{\partial \phi_{j}}{\partial n}-\phi_{j} \frac{\partial G_{i j}}{\partial n}\right) d s
$$

where $G_{i j}$ represents the Green function in matrix form that satisfies the Laplace equation for the entire fluid domain, and $\alpha$ is a solid angle ( $\alpha=0.5$ when singularities are on the boundary). For a two-dimensional problem, the simple source $G$ can be given by

$$
G\left(x_{i}, z_{i}, x_{j}, z_{j}\right)=-\frac{1}{2 \pi} \ln R
$$

where $R$ is the distance between the source $\left(x_{j}, z_{j}\right)$ and the field points $\left(x_{i}, z_{i}\right)$.

To update the dynamic and kinematic free-surface boundary conditions during the time simulation, the MEL method was adopted in order to describe the nonlinear free surfaces. Since the node velocity $(\vec{v})$ on the free surface was designed to move with the water particle velocity ( $\vec{v}=\nabla \phi$, full Lagrangian approach) and then apply the total time derivative, $\delta / \delta t=\partial / \partial t+\vec{v} \cdot \nabla$, the fully nonlinear free-surface conditions can be modified as follows:

$$
\frac{\delta \phi}{\delta t}=-g \eta-\frac{1}{2}|\nabla \phi|^{2}+\nabla \phi \cdot \vec{v}-\frac{P_{a}}{\rho}, \quad \frac{\delta \eta}{\delta t}=\frac{\partial \phi}{\partial z}-(\nabla \phi-\vec{v}) \cdot \nabla \eta \rightarrow \frac{\delta \vec{x}}{\delta t}=\nabla \phi
$$

where $\vec{x}$ is the node location $(x, z)$ and $P_{a}$ is zero. 
Then, by combining the artificial damping terms for the dissipation of the wave energy at the end of the domain, the free surface boundary conditions can be described as

$$
\frac{\delta \phi}{\delta t}=-g \eta+\frac{1}{2}|\nabla \phi|^{2}+\mu_{1}\left(\frac{\partial \phi}{\partial n}-\frac{\partial \phi^{*}}{\partial n}\right), \quad \frac{\delta \eta}{\delta t}=\frac{\partial \phi}{\partial z}+\mu_{2}\left(\eta-\eta^{*}\right)
$$

where $\mu_{1}, \mu_{2}$ denote damping coefficients. For the incident wave input, the reference values $\partial \phi / \partial n^{*}$ and $\eta^{*}$ were computed using the same computational condition as in the absence of bodies. The artificial damping scheme applied near the incident wave boundary is called frontal damping, and it serves to dissipate the re-reflection waves from the wave maker and preserve the incident waves for long-time simulation. For the forced-body input, the reference values were zero at both ends of the domain in order to dampen the radiated waves induced by the forced motion of the body.

A temporal ramp function at the incident wave boundary was applied in two wave periods $(2 T)$ to prevent the impulse-like behavior of the wave maker and to reduce the corresponding unnecessary transient waves. A spatial ramp function of two wavelengths ( $2 \lambda$ ) was also applied to the damping coefficients in Eq. (9) for efficient absorption of the propagating waves.

Energy loss due to viscosity occurs at the entrance of a chamber when the water surface moves up and down like a piston, especially for high velocity flows. The pressure drop at the chamber is generally proportional to the square of the water velocity. If the energy loss can be simplified to linearization of pressure drop, the flow velocity is proportional to vertical velocity of water column in the chamber, then the pressure drop can be described as

$$
P_{v}=K_{L}^{*} \overline{\dot{\eta}}=v \frac{\partial \phi}{\partial n}
$$

where $K_{L}^{*}$ and $\overline{\dot{\eta}}$ represent a linearized loss coefficient and a spatial-mean vertical surface velocity in the chamber, respectively. Moreover, $v$ denotes a linear viscous damping coefficient that can be determined by comparing the experimental results and the potential-fluid-based numerical results. Since the energy loss is related to the free surface elevation, the dynamic free surface boundary condition inside the chamber can be subsequently modified as

$$
\begin{array}{ll}
\frac{\delta \phi}{\delta t}=-g \eta-\frac{1}{2}|\nabla \phi|^{2}+\nabla \phi \cdot \vec{v}-\frac{1}{\rho} v \frac{\partial \phi}{\partial n} & \text { for linear viscous damping } \\
\frac{\delta \phi}{\delta t}=-g \eta-\frac{1}{2}|\nabla \phi|^{2}+\nabla \phi \cdot \vec{v}-\frac{1}{\rho} v_{q} \frac{\partial \phi}{\partial n}\left|\frac{\partial \phi}{\partial n}\right| & \text { for quadratic viscous damping }
\end{array}
$$

where $v_{q}$ denotes a quadratic viscous damping coefficient. More detailed numerical schemes and formulations used in this study, including the smoothing scheme and acceleration potential method, can be found in Koo and Kim (2004, 2010).

\section{NUMERICAL RESULTS AND DISCUSSIONS}

In order to analyze the hydrodynamic behavior of the free surface elevations of a floating body with an open chamber, numerical simulations were performed using body velocity inputs for forced-heaving bodies; the results are shown in Fig. 2. The triangles represent the experimental data of Faltinsen, Rognebakke and Timokham (2007), while the boxes denote the numerical results obtained using the present NWT. The dimensions of the floating body include a chamber gap of $0.18 \mathrm{~m}$, a body column thickness of $0.36 \mathrm{~m}$, and a water depth of $1.03 \mathrm{~m}$. The length of the free surface on each side of the domain is about four times the wavelength. After verification from a convergence test, 40 nodes on the free surface were chosen and a time step $(\Delta t)$ of $\mathrm{T} / 128$ was selected. 


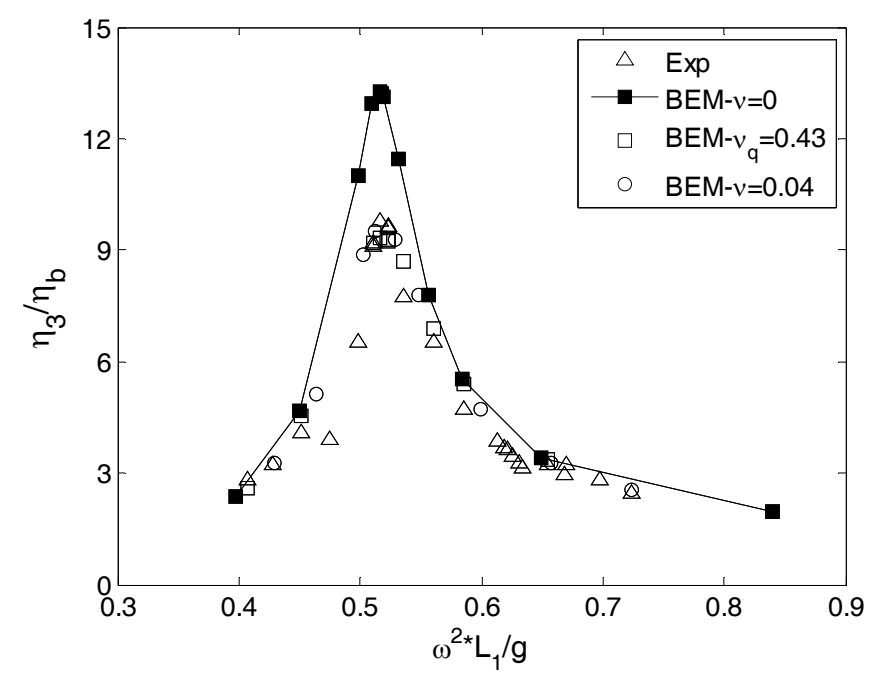

Fig. 2 Comparison of simulated mid-chamber free surface elevations with the experimental results; $\eta_{3}=$ mid-chamber free surface elevation, $\eta_{\mathrm{b}}=$ heaving body amplitude $(0.0025 \mathrm{~m}), \mathrm{L}_{1}=$ gap distance $(0.18 \mathrm{~m})$.

Since viscous energy loss occurred noticeably near the resonance frequencies, the numerical results without a viscous damping coefficient ( $v=0$, filled squares) show a deviation from the experimental data. However, with a tuned viscous damping coefficient ( $v=0.04$ for linear or $v_{q}=0.43$ for quadratic), the simulated results (unfilled squares or circle) agree well with the experiments. Note that viscous energy losses always occur when fluid passes between bodies; one of the critical areas for viscous loss in the present model was along the edge of the rectangular body. Thus, in the calculation based on the potential fluid, it was necessary to consider the viscous damping effect in order to obtain accurate results. For the present case, the quadratic viscous coefficient was optimized by comparison with experimental data, which was approximately 0.43 . The equivalent linear viscous damping coefficient $(v)$ of 0.04 was also selected as an optimal value.

Next, the effect of the corner shape of the body on the free surface elevation inside the chamber was investigated. The case of round edges on the submerged ends of the columns was calculated and compared with that of sharp edges, and the results are shown in Fig. 3. The filled marks (sharp edges) are defined exactly as in Fig. 2, whereas the unfilled marks are the results for the body with smooth edges. Since the present numerical results are based on potential theory (i.e., assuming an inviscid flow), the effect of vortex shedding, mainly due to the sharp edges, cannot be predicted. Consequently, the difference between the two numerical results from the inviscid fluid, which was caused only by the difference between the submerged areas of the body, was expected to be small.

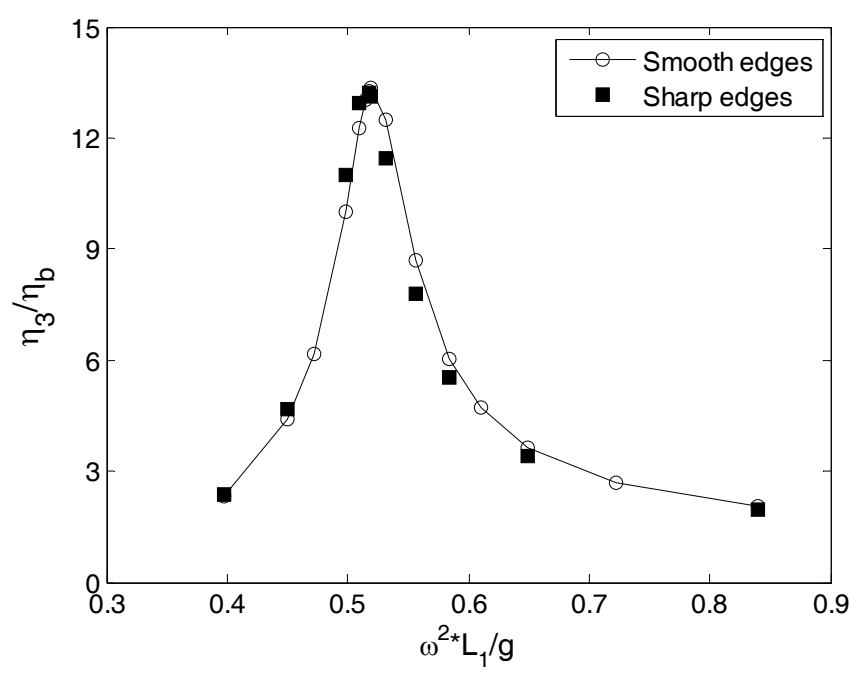

Fig. 3 Comparison of mid-chamber free surface elevation for different body edge shapes (sharp edges and smooth edges); corner radius $=0.01 \mathrm{~m}$, and no viscous coefficients were included. Other conditions are the same as Fig. 2. 
After verification of the calculated results, this numerical model was further extended to incorporate the two inputs: the forced-heave velocity of the body and the incident waves simultaneously. The analysis of this combined input system was vital in that the respective input parameters play particular roles in generating different surface elevations e.g., the frequency and magnitudes of the incident wave and body velocity, respectively, as well as the phase difference between the incident waves and radiated waves from the body motion. Accordingly, a comprehensive study was performed to investigate the specific effect of each input parameter on the resulting free surface elevations. Note that more attention was paid to the surface elevation inside the chamber, as the aim of this study was to find the optimum condition resulting in the greatest free surface elevation.

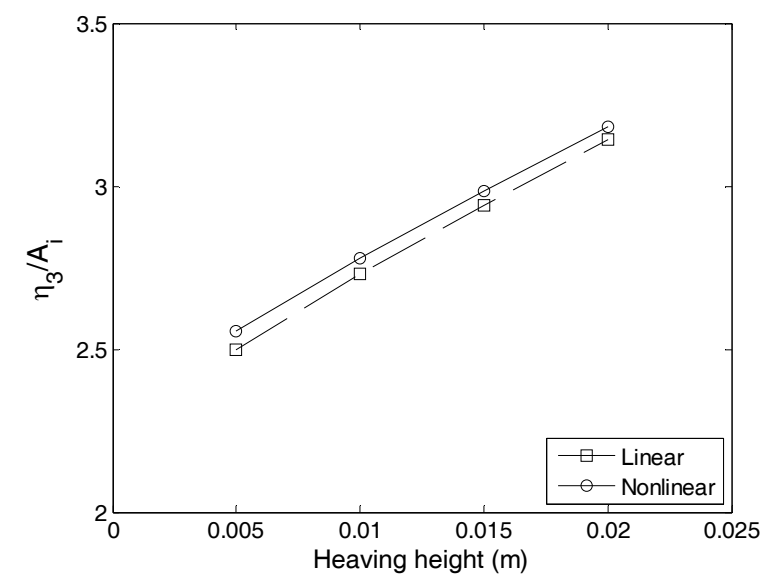

(a)

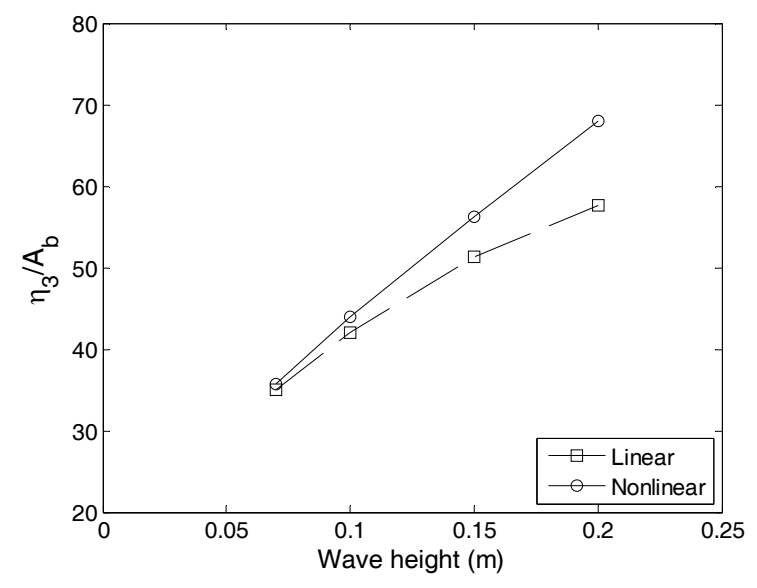

(b)

Fig. 4 Simulated mid-chamber free surface elevation (a) with respect to various heaving heights and a frequency $\left(\omega^{2} \mathrm{~L}_{1} / g\right)$ of 0.516 with a constant incident wave amplitude $\left(\mathrm{A}_{\mathrm{i}}=0.035 \mathrm{~m}\right)$ and a frequency $\left(\omega^{2} \mathrm{~L}_{1} / \mathrm{g}\right)$ of 0.498 , and

(b) with respect to various incident wave heights and a frequency $\left(\omega^{2} \mathrm{~L}_{1} / \mathrm{g}\right)$ of 0.498 with a constant heaving body amplitude $\left(\mathrm{A}_{\mathrm{b}}=\eta_{\mathrm{b}}=0.0025 \mathrm{~m}\right)$ and a frequency $\left(\omega^{2} \mathrm{~L}_{1} / \mathrm{g}\right)$ of $0.516 ; \eta_{3}=$ mid-chamber free surface elevation, $v_{q}=0.43$ (quadratic viscous damping coefficient).

Fig. 4 compares the mid-chamber free surface elevations after combining the two inputs for various heaving heights and incident wave amplitudes. With a constant incident wave input $\left(\mathrm{A}_{\mathrm{i}}=0.035 \mathrm{~m}\right)$ and a frequency $\left(\omega^{2} \mathrm{~L}_{1} / \mathrm{g}\right)$ of 0.498 , the surface elevations for various forced-heaving heights with a frequency $\left(\omega^{2} \mathrm{~L}_{1} / \mathrm{g}\right)$ of 0.516 are shown in Fig. $4(\mathrm{a})$. Note that as the heaving amplitude increases, the relative surface elevation gradually increases, which suggests that the magnitudes of the radiated waves also increases. In addition, the results of the linear and nonlinear calculations agree well, suggesting that the linear calculation for radiation problem of the forced-heaving body may be sufficiently accurate to predict the chamber surface elevation. Furthermore, these results indicate that the nonlinearities of the radiated waves inside the chamber are not significant in the high heaving body velocity.

Fig. 4(b) shows similar results when the forced-heaving input $\left(\eta_{b}=0.0025 m, \omega^{2} L_{1} / g=0.516\right)$ was kept constant while various incident wave heights with a frequency $\left(\omega^{2} \mathrm{~L}_{1} / \mathrm{g}\right)$ of 0.498 were given to the system. In this case, both the linear and nonlinear results increase with large incident wave heights, but there is a discrepancy between the two that grows as the wave height increases. This disparity can be explained by the nonlinear effects of the interacting waves, which can be properly demonstrated by fully nonlinear time domain simulation.

Fig. 5 presents the variation of the free surface elevation induced by a heaving body for various depth ratios in order to evaluate the effect of the sea bottom on the chamber free surface elevation. As the water depth increases, the relative free surface elevation increases. Moreover, the bottom effect becomes greater for small heaving amplitudes of the body; when the forced heaving amplitude is large, the effect of the sea bottom is clearly negligible. The nonlinear result for $\mathrm{H}_{\mathrm{b}}=0.03 \mathrm{~m}$ (where the heaving amplitude $\eta_{b}\left(=0.5 H_{b}\right)=0.015 \mathrm{~m}$ ) was also compared with the other linear calculations and was found to be nearly identical, affirming that the linear calculation is accurate enough to be used to calculate the wave radiation problem with a high heaving amplitude of a floating body.

For comparison with the case of the two inputs in Fig. 4, the chamber surface elevations with a single input are shown in 
Fig. 6. The linear and nonlinear results for various heaving body amplitudes are compared in Fig. 6(a) and are almost identical, indicating that the nonlinearity of the generated free surface elevation inside the chamber is not significant. Hence, the linear calculation is sufficiently accurate.

However, the linear and nonlinear results for various wave heights are different and the magnitude of deviation between them increases with high incident waves in Fig. 6(b). Consequently, it is expected that the magnitude of surface elevation is closely related to the nonlinearities of the incident wave heights, and in this case, the linear calculation is not sufficiently accurate for solving the diffraction problem in high incident waves.

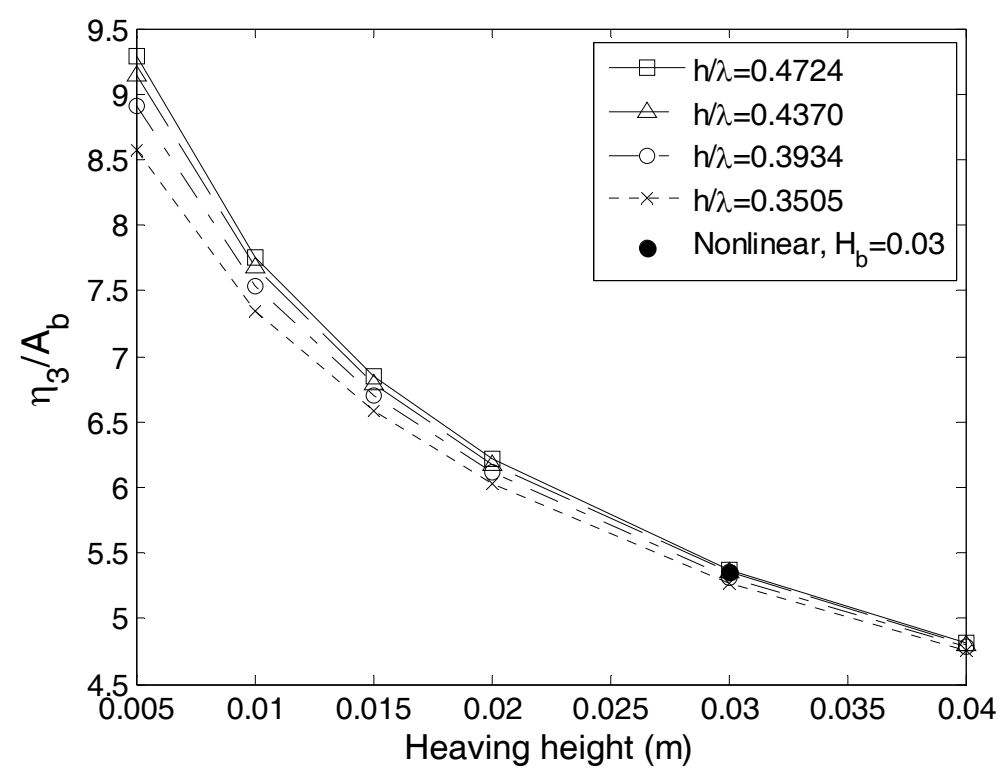

Fig. 5 Comparison of free surface elevations in the chamber for various heaving heights and motion frequency $\left(\omega^{2} \mathrm{~L}_{1} / \mathrm{g}\right)$ is 0.516 .

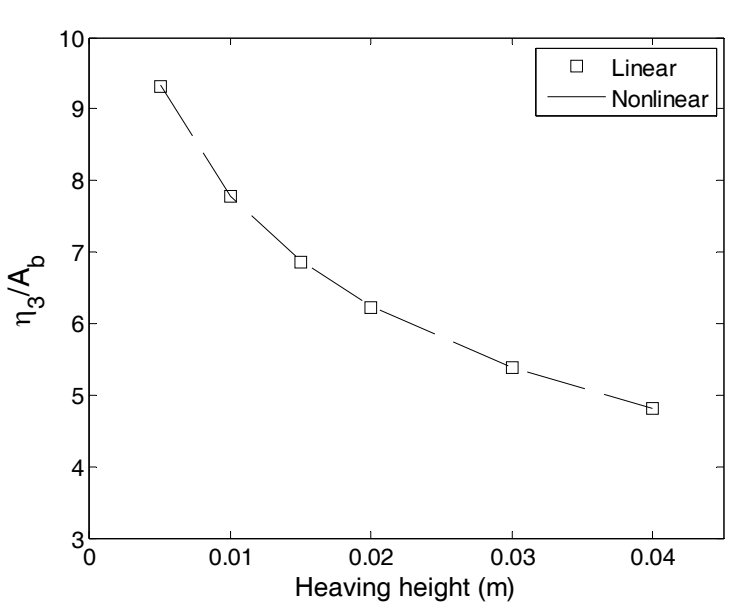

(a)

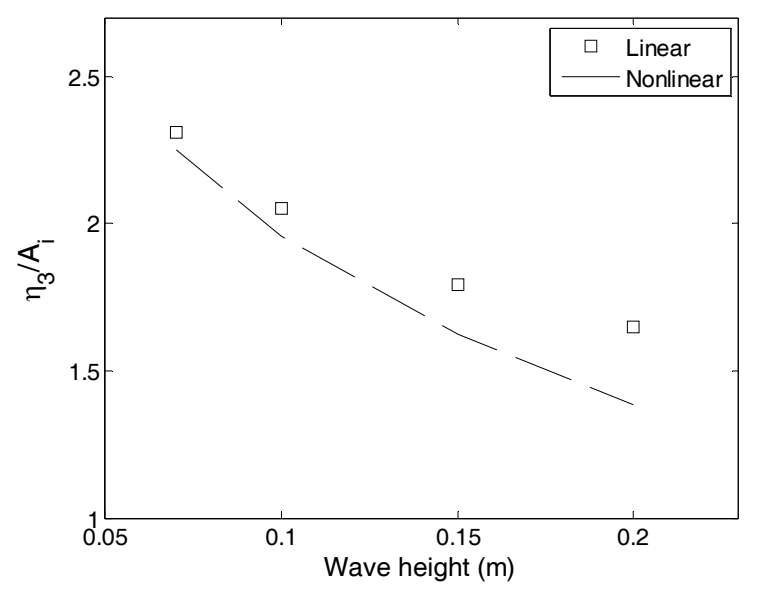

(b)

Fig. 6 Comparison of the linear and nonlinear results of mid-chamber free surface elevations for a single input system (a) with respect to the heaving height of the body $\left(\mathrm{H}_{\mathrm{b}}\right)$ with a frequency $\left(\omega^{2} \mathrm{~L}_{1} / \mathrm{g}\right)$ of 0.516 , and

(b) with respect to the incident wave height $\left(\mathrm{A}_{\mathrm{i}}\right)$ with a frequency $\left(\omega^{2} \mathrm{~L}_{1} / \mathrm{g}\right)$ of 0.498 .

Thus, the case of a large heaving height $\left(\mathrm{H}_{\mathrm{b}}=0.02 \mathrm{~m}\right)$ was simulated in the linear time domain and compared to the results of the $\mathrm{H}_{\mathrm{b}}=0.005 \mathrm{~m}$ case. Fig. 7 highlights a distinct difference between the two cases that grows with increasing wave height. Moreover, it is noteworthy that the relative surface elevation for a small heaving height is greater than that for a large heaving height. In other words, the increasing rate of relative surface elevation for various wave heights is greater for small heaving 
amplitudes, which suggests that greater forced heaving amplitudes deteriorate the surface elevation in the chamber induced by higher incident waves.

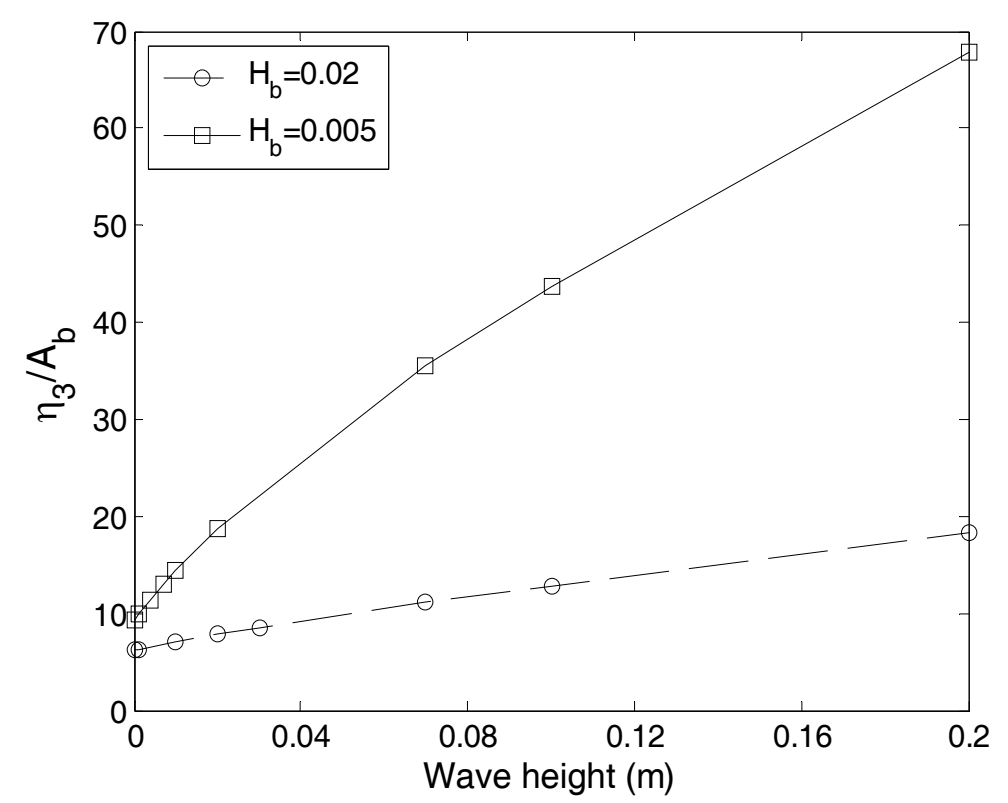

Fig. 7 Simulated mid-chamber free surface elevation with respect to wave height for two different heaving inputs $\left(\mathrm{H}_{\mathrm{b}}=2 \mathrm{~A}_{\mathrm{b}}\right)$. The heaving frequency $\left(\omega^{2} \mathrm{~L}_{1} / \mathrm{g}\right)$ is 0.516 and the wave frequency $\left(\omega^{2} \mathrm{~L}_{1} / \mathrm{g}\right)$ is 0.498 .

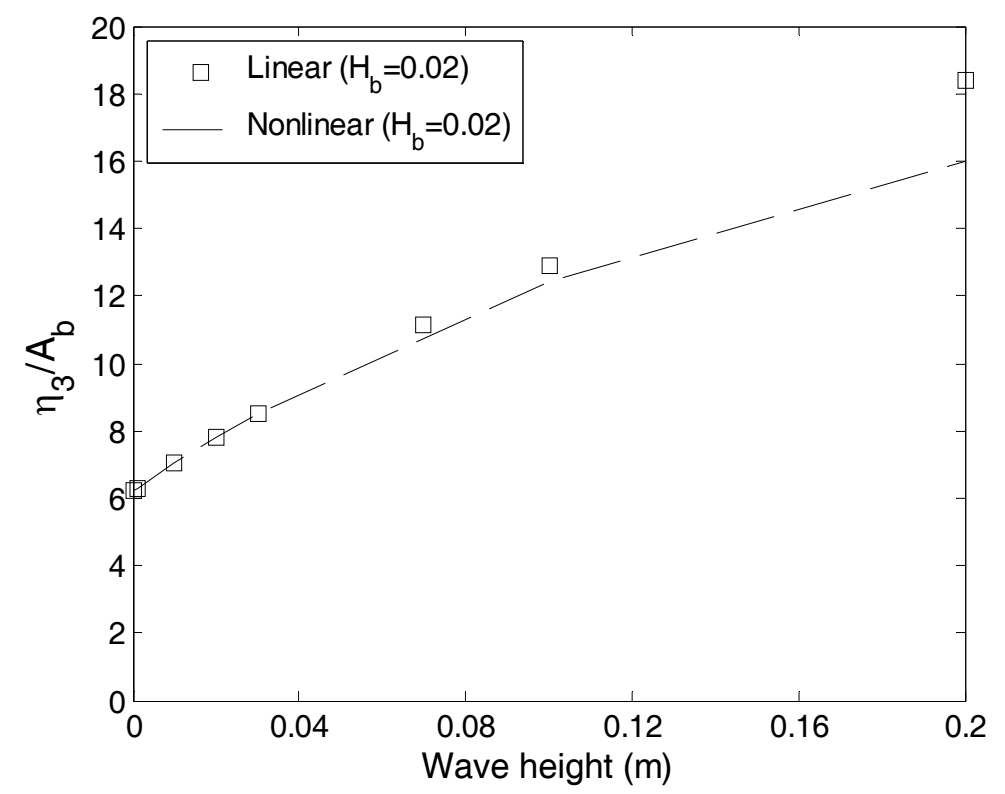

Fig. 8 Simulated mid-chamber free surface elevations for linear and nonlinear calculations with a heaving height $\left(\mathrm{H}_{\mathrm{b}}\right)$ of $0.02 \mathrm{~m}$ and a frequency $\left(\omega^{2} \mathrm{~L}_{1} / \mathrm{g}\right)$ of 0.516 . Incident wave frequency $\left(\omega^{2} \mathrm{~L}_{1} / \mathrm{g}\right)$ is 0.498 .

Fig. 8 shows the comparison of surface elevations of the linear and nonlinear calculations for various wave heights with a given heaving height $\left(\mathrm{H}_{b}=0.02 \mathrm{~m}\right)$ and a heaving frequency $\left(\omega^{2} \mathrm{~L}_{1} / \mathrm{g}=0.516\right)$. In particular, it shows that the relative surface elevation of the linear calculation was overestimated for high incident wave heights, which is similar behavior to the single input case shown in Fig. 6(b). 


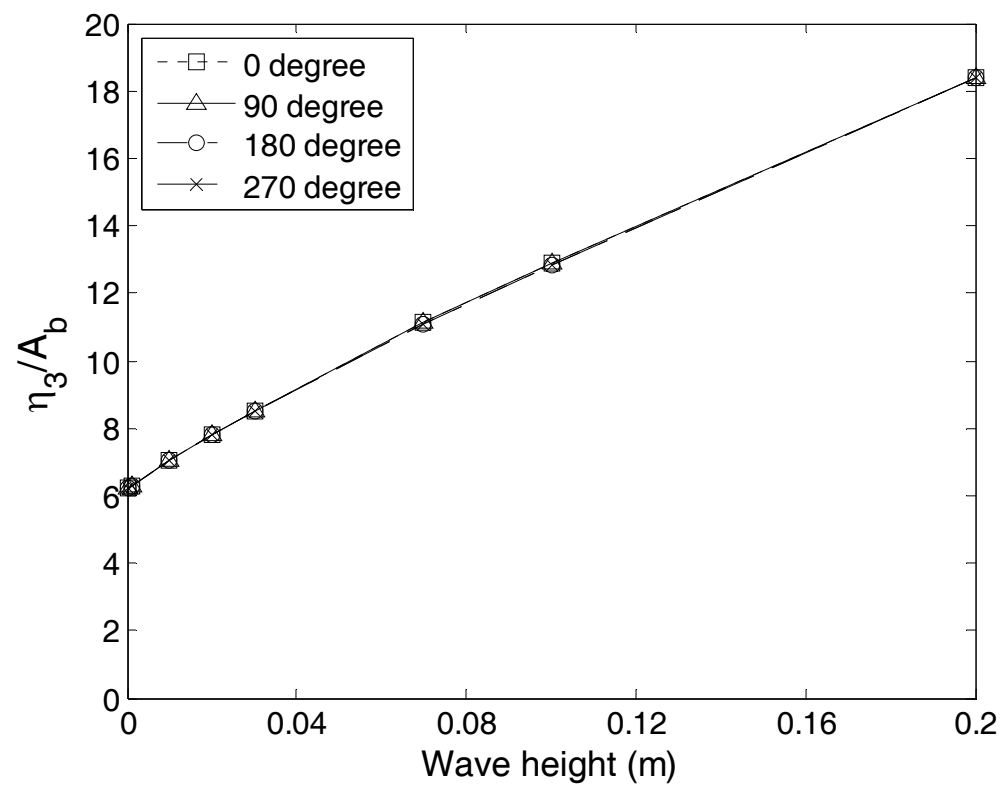

Fig. 9 Comparison of simulated mid-chamber free surface elevation for four different phase angles, given a forced-heave motion of the body $\left(\mathrm{H}_{\mathrm{b}}=0.02 \mathrm{~m}\right)$ and a frequency $\left(\omega^{2} \mathrm{~L}_{1} / \mathrm{g}\right)$ of 0.516 .

Incident wave frequency $\left(\omega^{2} \mathrm{~L}_{1} / \mathrm{g}\right)$ is 0.498 .

Finally, in order to evaluate the effect of the phase difference between the radiated waves from forced heaving and the incident waves, surface elevations for various phase angles are compared in Fig. 9. These results show that the effect of the phase angle on the mid-chamber elevation is negligible when the maximum surface elevation is obtained in the two-input system.

\section{CONCLUSIONS}

Hydrodynamic analysis of a surface-piercing body with an open chamber was performed with a two-input system including regular incident waves and forced-heaving body motions. The floating body was simulated in the time domain using a fully nonlinear 2D NWT technique based on potential theory, BEM with constant panels, an MEL approach for considering the nonlinear free surfaces, and an RK4 time-integration scheme. This paper mainly analyzed the hydrodynamic behavior of the free surface inside the chamber for various input conditions and evaluated these respective inputs. The viscous effect on the free surface elevation, representing vortex shedding at the body edge, was appended to the calculation after the comparison with experimental data. It was found that the linear calculation for the radiation problem of a forced-heaving body was sufficiently accurate to predict the chamber surface elevation. In addition, the magnitude of the surface elevation in the chamber was found to be closely related to the nonlinearities of incident wave heights, and the linear calculation was not able to produce a satisfactory solution for the wave diffraction problem. However, the fully nonlinear calculation was able to properly demonstrate the nonlinear effects of interacting waves inside the chamber. Also note that the effect of the phase difference between the two inputs was not significant on the resulting free surface elevations inside the open chamber.

\section{ACKNOWLEDGEMENTS}

This work was supported by the 2010 SOTOP Research Fund of University of Ulsan.

\section{REFERENCES}

Faltinsen, O.M., Rognebakke, O.F. and Timokham, A.N., 2007. Two-dimensional resonant piston-like sloshing in a Moon pool. Journal of Fluid Mechanics, 575, pp.359-397. 
Heath, T., Whittaker, T.J.T. and Boake, C.B., 2000. The design, construction and operation of the LIMPET wave energy converter (Islay, Scotland). Proceedings of $4^{\text {th }}$ European wave energy conference. Aalborg Univ, Denmark. pp.49-55.

Koo, W. and Kim, M.H., 2004. Freely floating-body simulation by a 2D fully nonlinear numerical wave tank. Ocean Engineering, 31(16), pp.2011-2046.

Koo, W.C., 2009. Nonlinear time-domain analysis of motion-restrained pneumatic floating breakwater. Ocean Engineering, 36(9-10), pp.723-731.

Koo, W.C. and Kim, M.H., 2010. Nonlinear time-domain simulation of a land-based oscillating water column (OWC). Journal of Waterway, Port, Coastal and Ocean Engineering, 136(5), pp.276-285.

Lin, W.M., Newman, J.N. and Yue, D.K.P., 1984. Nonlinear forced motion of floating bodies. Proceedings of $15^{\text {th }}$ Symposium Naval Hydrodynamics. Hamburg, Germany. pp.33-49.

Masuda, Y. and Miyazaki, T., 1978. Wave power electric generation study in Japan. International Symposium on wave and tidal energy, British hydromechanics research association fluid engineering. Cranfield, Beds., England 27-29 September 1978.

Yong, L. and Mian, L., 2010. Wave-body interactions for a surface-piercing body in water of finite depth. Journal of Hydrodynamics, 22(6), pp.745-752. 